\title{
EDUCACIÓN Y FILOSOFÍA. UN ABORDAJE A PARTIR DEL CONCEPTO DE "PENSAMIENTO" DE ALAIN BADIOU
}

\author{
Leonardo Javier Colella*
}

\begin{abstract}
Resumen
El presente artículo busca reconstruir una perspectiva filosófica de la educación a partir de las nociones de "pensamiento" y "filosofía" de Alain Badiou. El autor no teoriza sobre la educación, sin embargo, intentaremos aplicar sus conceptos de "saberes enciclopédicos" y "verdades" al ámbito educativo para realizar una crítica a la circulación de saberes y caracterizar una educación que incluya las nociones de "pensamiento" y "verdad".
\end{abstract}

Palabras clave: Educación y filosofía. Pensamiento. Verdad.

\begin{abstract}
This paper aims to reconstruct a philosophical perspective on education from the notions of "thought" and "philosophy" of Alain Badiou. The author does not theorizes on education, however, we'll try to apply his concepts of "encyclopedic knowledge" and "truth" to the educational environment for a critique of the circulation of knowledge, and characterize an education that includes the notion of "thought" and "truth".
\end{abstract}

Keywords: Education and philosophy. Thought .Truth.

\section{El pensamiento}

Badiou utiliza el concepto de "pensamiento" para caracterizar la toma de posición subjetiva frente a los saberes preestablecidos. Pensar sería, en

\footnotetext{
* Becario doctoral del Consejo Nacional Científico y Técnico (CONICET). Profesor Universitario de Filosofia por la Universidad Nacional de General Sarmiento (UNGS) Argentina. E-mail: leonardojcolella@yahoo.com.br
} 
este sentido, interceder, atravesar y reconstruir los saberes que enuncian $l o$ que hay. El pensamiento constituiría, para Badiou, una ruptura con respecto a los saberes enciclopédicos que representan un estado de cosas dado ${ }^{1}$. Es la desvinculación cuantitativa entre un múltiple infinito y la parte que lo contiene la que abre, en tanto impronunciable (lo-imposible-de-decir), la perspectiva de elecciones ilimitadas.

El pensamiento es la prueba de la no-resignación del ser a la presión representativa de las partes: "Un pensamiento no es otra cosa que el deseo de poner fin al exorbitante exceso del estado" (BADIOU, 1988, p. 314). Y el portador de ese deseo de pensar asume una forma subjetiva.

Sin embargo, existe la idea bastante extendida, para el ámbito teórico de la educación, de asociar la presentación de los elementos de una situación con su nominación. Esta perspectiva elude la distinción entre la situación (aquella que presenta las multiplicidades infinitas que son los individuos miembros de una situación compleja y aleatoria) y su estado (que incluye esas multiplicidades a las que agrupa como partes y nomina como "estudiantes", "docentes", etc.). Es decir, se trata de la creencia de que los múltiples presentados son tales por poseer "nombres": ser presentado o ser nombrado sería indistinto.

En aquel sentido, todo término existente en una situación será tal si está nombrado. La lengua es el elemento que asimila sin ambigüedad al múltiple presentado con la parte que lo representa. El estado dirige la lengua y ordena la representación de los elementos.

Este estrecho vínculo entre una parte y los elementos de una situación, esa proximidad establecida por la lengua, que opera agrupando aquellos múltiples que poseen alguna propiedad común y distinguiéndolos de los demás, permite que el estado controle la situación, que el exceso del vacío sea controlable. El saber de una situación es quien mantiene la proximidad entre la pertenencia y la inclusión, es quien mantiene reglada esta distancia.

Un múltiple presentado en situación, indiscernible para la cuenta me-

1 Un concepto central de la obra de Alain Badiou es el de "pensamiento". Como mencionamos, este término es empleado por el autor de forma radical y vinculado a una disrupción. Esta radicalidad como carácter definitorio del pensar es abordada por Boostels (2002) en "Vérité et forçage: Badiou avec Heidegger et Lacan". Asimismo, para una relación del concepto de "pensamiento" en Badiou y Deleuze, puede verse Bergen (2002), "Pensée et Etre chez Deleuze et Badiou (Badiou lecteur de Deleuze)". 
taestructural, será ajeno al saber y, por tanto, inexistente para él. De este modo, el estado prescribe, mediante el saber, la existencia de los términos. La lógica del saber expulsa fuera de sí todo pensamiento y, con él, toda dialéctica del acontecimiento y de la intervención subjetiva.

Sólo construye saberes reglados propios de la situación pero no participa de las decisiones y de las invenciones que atañen a las disrupciones del estado que la sustenta. El cambio, para esta lógica, no es otra cosa que las modificaciones regulares y las construcciones organizadas de los saberes de sus partes. Estas modificaciones son las que impulsan, en el ámbito educativo, las actualizaciones curriculares. Éstas no responden a otra cosa más que al destino de un camino ya prefigurado, por caso, a los avances técnicos, a las nuevas tecnologías, a las modas, a nuevos dispositivos de control y, en sus versiones más progresistas, al intento por explicitar las reglas de juego, por una inclusión en los devenires forzados del mundo contemporáneo. El saber responde a la sociedad del conocimiento, que se halla bastante distanciada de lo que, con Badiou, podríamos denominar sociedad del pensamiento.

Todo aquello que no es susceptible de ser clasificado en un saber, no-es. La ontología badiouiana impugna el saber en tanto enfatiza la potencia del vacío, mientras que su lógica (la del propio saber) domina todo exceso a través de la nominación y el ordenamiento de las partes designadas por el estado. Como señalaremos más adelante, el saber cuenta con una característica sustancial, su transmisibilidad. Mientras que el "saber" es transmisible, el "pensamiento" exige su actualización, individual y colectiva.

Badiou afirma que la creencia más extendida actualmente es la que asevera que "no hay más que cuerpos y lenguajes" (BADIOU, 2006, p. 17). Lo realmente existente son los cuerpos, y con él, la finitud, el deseo, el goce, el sufrimiento y la muerte. Propone el nombre de "materialismo democrático" para la posición que sostiene ese axioma. Este materialismo (o biomaterialismo) se preocupa, antes que nada, por la defensa de la vida biológica. Así, los derechos "humanos" serán los derechos que apunten a la conservación y al desarrollo de la vida: la protección humanista de los cuerpos vivientes.

Al admitir la existencia, además de los cuerpos, de una diversidad de lenguajes, el materialismo se presenta como "democrático" ya que requiere del consenso y de la igualdad jurídica para tratar aquella pluralidad. La humanidad pierde así su especificidad (que ya hemos mencionado que para 
Badiou era más bien el "pensamiento" o la "Idea"), para ser absorbida por la generalidad biológica.

La propuesta del propio Badiou es la de trastocar, agregando un tercer término, el axioma del materialismo democrático: "no hay más que cuerpos y lenguajes, sino que hay verdades". Las verdades aquí no serían ni una adición ni una síntesis de los dos primeros términos (cuerpos y lenguajes). La expresión, gramaticalmente forzada, intenta denotar que ciertamente existen sólo cuerpos y lenguajes, pero que no sólo hay "lo que hay", sino que existen las verdades como excepciones a lo que hay. Badiou reconoce que la estructura lógica de los mundos se halla compuesta por cuerpos y lenguajes, pero asume a la verdad como el nombre filosófico de aquello producido en las disrupciones del orden lógico de esos mundos.

Badiou propone, de forma crítica, una variable antropológica para el materialismo democrático, "no hay más que individuos y comunidades", y junto a ella, advierte un cúmulo de significantes propagandísticos: "respeto por el otro", "diversidad cultural", "pluralidad de ideas", etc. Su variante educativa asume este axioma, según el cual no hay más que individuos y naciones, para construir una educación de estado. La educación por verdades de la dialéctica materialista se opone a ello postulando un sujeto que se sustrae a toda individuación y a todo nacionalismo, un cuerpo subjetivo que no es ni individual ni nacional, sino colectivo y universal.

Con el término verdad, Badiou intenta designar la existencia de algo que no se halle históricamente determinado, que pueda "aparecer" de forma distinta en diversos mundos, incluso con siglos de distancia: podríamos pensar aquí, por ejemplo, aquello que han presentado o presentan "en común" la propuesta jacotista de principios de siglo XVIII, la tesis de la autoeducación de las masas en China de mediados del siglo anterior, las revueltas antijerárquicas del Mayo francés, y las más actuales experiencias de educación zapatista o de la Argentina post 2001 (bachilleratos populares y seminarios autogestionados). La máxima de la filosofía, para Badiou, es la igualdad absoluta, todo lo que se ubique en una tendencia inversa permanece extranjero a toda verdad:

uno de mis propósitos ha sido transformar la noción de verdad de manera que ella obedezca a ese mandato [la igualdad]. Las cosas marchan también en ese sentido: transformar la noción de verdad de manera que ella obedezca a la máxima igualitaria (BADIOU, 2004, p. 63). 
La verdad en Badiou cuenta con tres características principales: depende de un surgimiento, de un acontecimiento; es radicalmente universal, está constituida por el "para-todos" igualitario puro y genérico; no es consecuencia del sujeto, sino que es constitutiva de éste, o en todo caso, la verdad es desplegada junto con el proceso de fidelidad militante de éste. Veamos a continuación cómo las verdades se distinguen de los saberes.

\section{Saber, verdad y enciclopedia}

Badiou utiliza el término "genérico" para referirse a aquello que es indiscernible por estar sustraído del saber o de la nominación de una situación. "Genérico" es sinónimo de "indiscernible", pero ambos difieren en que este último posee una connotación negativa (en cuanto refiere a aquello no-discernible) mientras el primero intenta adquirir una evocación propositiva, en cuanto alude a la verdad misma de una situación: “'Genérico' pone en evidencia la función de verdad de lo indiscernible. La negación implicada en 'indiscernible' conserva, sin embargo, algo esencial: que una verdad es siempre lo que agujerea un saber" (BADIOU, 1988, p. 363).

La construcción de un sujeto ligado a la educación, entonces, se vinculará con la dupla verdad-saber. En efecto, los procesos de construcciones subjetivas estarán ligados a la tensión desplegada entre un pensamiento postacontecimiental y los saberes metaestructurales prefijados que Badiou denomina "enciclopedia".

El saber, como señalamos anteriormente, denota la capacidad de discernir y clasificar los múltiples de una situación según determinadas propiedades y atributos. Todo dominio de saber actúa de acuerdo a dos operaciones básicas: el discernimiento, en tanto capacidad para extraer las propiedades de un término (y que se opone a la genericidad de las verdades) y la clasificación, en tanto facultad de segmentación y agrupamiento (vinculada con las "partes", y en consecuencia, con el estado). Este es el modo en que opera la enciclopedia.

El saber le otorga a un múltiple un determinante enciclopédico que hace que ese elemento pertenezca a una "parte". Incluso es posible que un mismo término sea alcanzado por diversos determinantes (tal sería el caso de un individuo de un encuentro educativo juzgado por la propiedad "posesión de ciertos contenidos" y nominado "profesor", y el mismo individuo juzgado, 
desde otro determinante, por el grado de conocimientos y habilidades que aún no posee, y denominado, por ejemplo, "estudiante de posgrado", etc.).

El saber, afirmamos previamente, ignora el acontecimiento y sus consecuencias, ya que éste sobrepasa el lenguaje de la situación. En la educación, el tema del saber podría pensarse en dos sentidos. El primero de ellos, refiere a los contenidos puestos en juego en una situación educativa (sean estos la razón fundacional de todo acto educativo, como en el caso de la lógica institucionalizada, o sean considerados más lateralmente, como insumos que confrontan con el pensamiento, en el proceder de experiencias educativas alternativas). Se opondrían así, una educación que busca transmitir saberes en tanto transferencia de los conocimientos que enuncian un estado dado de cosas en una ciencia particular, o una educación que propone la participación en el pensamiento producto de las novedades acontecidas en diversos campos. Esta distinción no refiere a otra cosa más que a la oposición entre una educación "estatal" y una educación "por verdades", planteada en casi la totalidad de las propuestas educativas que adoptan el marco conceptual de Alain Badiou.

Entre ellos, podemos destacar los trabajos de A. J. Bartlett, quien busca utilizar diversos conceptos badiouianos para desplegarlos en el ámbito educativo: "Conditional notes on a New Republic" (2006a), "The pedagogical theme: Alain Badiou and an eventless education" (2006b) y Badiou and Plato. An education by truths (2011). Para el autor, es indispensable referir la propuesta filosófica de Alain Badiou al pensamiento de Platón. Su tesis central sostiene que ambos concuerdan en concebir a la filosofía como una "educación por verdades" que se contrapone con una "educación estatal". Esta última sólo considera la puesta en juego de saberes enciclopédicos mediante los que se tiene en cuenta cada elemento de una situación (en tanto pertenecientes a una "parte").

El segundo sentido del saber en la educación es el del saber pedagógico. Se trata de indagar bajo qué condiciones se puede (y quiénes pueden) pensar aquello que ocurre en las situaciones educativas. Para la educación cuya lógica es la explicación, la asignación de los determinantes enciclopédicos, el estudio sobre las relaciones de los integrantes de un encuentro educativo, las propiedades atribuidas a cada uno de ellos, su clasificación, su nominación y demás cuestiones, quedan relegadas a un tipo de saber: el saber pedagógico. 
Éste es el saber del estado de la situación, pero que paradójicamente, es extraído de las situaciones particulares para erigirse en un campo de estudio, cuyos especialistas son ajenos a esos encuentros singulares. No se trata aquí de la extendida acusación contra investigadores o funcionarios de la educación que, por las presiones de la productividad del conocimiento o de la gestión política, dedican su vida profesional por fuera de la práctica docente, sino de que se traza una distancia irreductible entre lo que está sucediendo en un determinado encuentro educativo (quiénes son tenidos en cuenta, según qué criterios, bajo qué principios, etc.) y los propios protagonistas de ese encuentro (sus perspectivas, sus vivencias, sus pensamientos, sus decisiones), generando una especie de inercia pedagógica que obturaría cualquier proceso de subjetivación colectiva. Lo habitual en las situaciones educativas es que sus propios protagonistas no "piensen" sobre lo que está ocurriendo en la propia situación (sobre qué determinantes enciclopédicos los definen, sobre qué esquemas políticos son atravesados, sobre qué tipos de subjetivación son contenidos, etc.).

En este último sentido, una educación por verdades sería también aquella permeable a las disrupciones ocurridas en las situaciones educativas y que traerían consigo la posibilidad de nuevas nominaciones, nuevas relaciones, nuevas figuras subjetivas, sobre el propio encuentro educativo y más allá del saber pedagógico reglado por el estado. Sin embargo, este último sentido se halla ausente en casi la totalidad de las propuestas teóricas sobre educación desde una perspectiva badiouiana.

Señalamos anteriormente que una verdad era indiscernible e inclasificable para la enciclopedia. El acontecimiento no puede ser subsumido en el lenguaje del saber. El nombre del acontecimiento, su declaración, puede estar enunciado a través de sencillos signos del saber (e.g., "hay que reinventar la educación"). Sin embargo, éstos no escapan de la enciclopedia en tanto términos o significantes, sino que lo hacen en tanto nombres del acontecimiento, manifestaciones del vacío, forcluidos del saber.

Una verdad evita todo determinante de la enciclopedia, aquellos múltiples ligados a esa verdad serán indiscernibles e inclasificables para el saber. Los procesos de subjetivación en la educación no están ligados al saber, sino al cambio que hace posible una nueva existencia: "Una fidelidad no puede depender del saber. No se trata de un trabajo sapiente sino de un trabajo militante" (BADIOU, 1988, p. 365). El procedimiento fiel "en modo alguno está predeterminado por el saber. Su origen es el acontecimiento, que el saber 
ignora..." (BADIOU, 1988, p. 374). En tal sentido, los procesos subjetivos no están vinculados al saber enciclopédico sino, más bien, al pensamiento creativo requerido por un acontecimiento que evita el lenguaje prestablecido.

Un procedimiento genérico supone ciertos múltiples que no pueden ser clasificados por ningún determinante enciclopédico y que, en consecuencia, son innombrables de acuerdo al lenguaje del saber propio del estado de la situación o del Estado. Esos múltiples son interpelados por una verdad al fulgor de un acontecimiento. Ahora bien, ya hemos afirmado la distinción entre la verdad y el saber.

Del mismo modo que para la educación explicadora la exigencia se halla en relación al saber, para la educación emancipatoria la exigencia ocurre respecto del pensamiento o de las verdades. ¿Qué podría significar, por ejemplo, para el ámbito de la enseñanza científica, que una educación prescinda de los saberes que, en apariencia, son el fundamento y la esencia de esas ciencias? Una educación cuya exigencia es el pensamiento, con la carga conceptual que le hemos asignado a este último, interpretaría a la ciencia en su ser infinito, esto es, como un procedimiento de invención y no como sus fragmentarios resultados.

Entonces, una educación cuya exigencia es el "pensar" requiere intervenir en la situación en la que un acontecimiento ha tenido lugar justamente "pensando" la situación a partir del suplemento disruptivo que impulsará a crear una nueva forma de nombrar las novedades, y a partir de las consecuencias que esto significa respecto de los saberes previos. Este acto creativo del pensamiento en la educación puede referir a dos cuestiones: a las verdades producidas en los diversos campos (que, como sugeriremos más adelante, la vincularía directamente con la filosofía) o a las verdades políticas, amorosas, artísticas o científicas producidas en el propio encuentro educativo.

Estas observaciones respecto de la educación están vinculadas a la consideración de los procesos subjetivos que en ella se desplieguen. Si afirmamos que la propiedad fundamental de los saberes es su transmisibilidad, entonces, una educación centrada en la explicación (o en la circulación) de saberes destinará su atención a los procesos subjetivos de orden cognitivo, didáctico, sociológico, etc. de forma individual o plural (con preeminencia en la "unidad" que aprende o que enseña). Por el contrario, la exigencia del pensamiento (i.e., más allá del saber) hace que la actividad sustancial de la educación por verdades no asuma la forma de una explicación escolar, sino 
más bien la del ejercicio del pensar. Este ejercicio es el de un pensar colectivo debido a la exigencia del postulado igualitario, que requiere siempre la verificación de una potencia universal. A consecuencia de ello, sus procesos de subjetivación deben ser asumidos también de forma colectiva y trascender el universo de los saberes, de su incorporación y de su transferencia.

\section{Filosofía y educación}

En un artículo denominado "Philosophy as Creative Repetition" (2007), Alain Badiou propone describir a la filosofía como una forma de "repetición creativa". En ella distingue esencialmente dos tendencias acerca de la identidad de la filosofía. La primera de ellas la interpreta como un saber reflexivo: un conjunto de conocimientos sobre la verdad y los valores en los ámbitos teoréticos y prácticos, respectivamente. La forma que despliega el desarrollo de esta tendencia es la de la explicación escolar, entendida como método de transmisión de aquellos saberes reflexivos. La segunda postura concibe a la filosofía como una acción: una transformación subjetiva, una conversión radical. En este caso, la filosofía es delimitada por la singularidad de un acto, que Badiou asocia con la acusación ejercida contra Sócrates de "corromper a los jóvenes", cuyo rasgo distintivo sería impulsar el ejercicio de la crítica y la revuelta, por sobre el de la imitación y la obediencia. Esta segunda tendencia, que interpreta a la filosofía como una actitud antes que como un conocimiento, no se enseña mediante la explicación escolar, sino que se vive o se experimenta con otros, al modo en que Sócrates dialogaba con los jóvenes en las calles de Atenas.

En este sentido, la idea de "repetición creativa", aplicada al desarrollo de la filosofía, describe la existencia de un acto que es formalmente común a todas las corrientes filosóficas. En éstas, lo invariable sería el gesto de división que constituye el acto filosófico: la distinción con respecto a otras filosofías y demás saberes; y la reorganización de las experiencias teoréticas y prácticas a partir de esas nuevas divisiones normativas. La filosofía se hallaría delimitada por el insistente dinamismo entre lo que afirma y lo que cuestiona, por la relación tensional entre un ejercicio crítico de afirmación, oposición y creación. A diferencia de la ciencia, en la que sus propias novedades reemplazan las teorías anteriores, ella se constituye a partir de ese permanente afirmar y refutar. Según Badiou, en la filosofía siempre existirá 
una intención de reconstituir lo existente partiendo de disposiciones normativas nuevas: se trata de poner de relieve el lazo entre el ser y el acontecer, lo que conforma el rasgo propio de la "repetición creativa".

Este gesto crítico, que se reitera a través de los diferentes filósofos, se lleva a cabo bajo determinadas condiciones variables: las transformaciones propias de los campos acontecimientales. Cuando el acto filosófico se enfrenta a la actualidad específica de cada contexto se produce una nueva creación intelectual. El acto filosófico se ejerce, según Badiou, luego del despliegue de algunos acontecimientos en política, ciencia, arte y amor. Estos acontecimientos son su condición de posibilidad. En tal sentido, el autor afirma que la filosofía dependería de algunos campos no-filosóficos que serían sus "condiciones".

Recapitulando, lo que hay de repetición en la filosofía es el gesto crítico del acto filosófico, y lo que hay de creación es impulsado por el nuevo contexto específico en el que se desarrolla dicho acto. Es por eso que el autor sugiere el concepto de "repetición creativa" para definir a la filosofía.

En Conditions (1992), Badiou afirma como primer punto para el diagnóstico de la filosofía, que ésta se encuentra "paralizada" por su propia historia: "la filosofía no es ya justamente sino su propia historia; ella deviene el museo de sí misma. Llamo parálisis de la filosofía a ese vaivén entre la historiografía y la desubicación" (BADIOU, 1992, p. 51). Y luego del diagnóstico, el autor propone romper con aquel historicismo. En este sentido, reafirma a la filosofía como una actitud sobre el presente más que como un cúmulo de saberes específicos, por más generales y abstractos que se pretendan. La historia de la filosofía vendría a refrenar la auténtica tarea de la filosofía:

la presentación filosófica debe autodeterminarse inicialmente sin referencia a su historia. Debe tener la audacia de presentar sus conceptos sin hacerlos previamente comparecer ante el tribunal de su momento histórico. (...) La filosofía debe autodeterminarse de tal suerte que sea ella la que juzgue su historia, y no su historia la que la juzgue a ella (BADIOU, 1992, p. 52-53).

Para ensayar una definición de la filosofía, Badiou procura distinguirla de lo que él denomina la sofística moderna (asociada a las convenciones, 
géneros y sentidos plurales del discurso), e intenta ligarla al concepto de "verdad". Por ello la categoría de verdad será central en la propia tarea filosófica: "La filosofía es una construcción de pensamiento donde se proclama, contra la sofística, que hay verdades" (BADIOU, 1992, p. 58).

¿Qué rasgos comunes podríamos hallar entre la filosofía y la educación, tal como las propone pensar Alain Badiou? La filosofía, del mismo modo que la educación, no es "productora" de verdades, sin embargo, "opera" con las verdades producidas en los diversos campos (política, ciencia, arte y amor). La filosofía al igual que la educación, entonces, trabaja sobre las verdades que le son externas. En consecuencia, no constituyen en sí mismas un saber, aunque éste se halle implicado en sus operaciones. Badiou señala que la operación sustancial de la filosofía es la "captura".

La filosofía captura, mediante la actividad del pensamiento, las verdades de los diversos campos, sin por ello establecer ninguna relación jerárquica o de fundamento entre ambos. La tarea de la filosofía es "mostrar" las verdades, esto es, distinguirlas del ámbito de la opinión (BADIOU, 1998, p. 60). Cuando la filosofía se asume como productora de verdades, y ya no como un aparato de "captación", es cuando sobreviene lo que Badiou denomina "el desastre de la sutura". En efecto, para Badiou, la ciencia, la política, el arte y el amor son "condiciones" de la filosofía. El hecho de ser condiciones no significa que sean "fundamentos" de ella. Cuando alguno de estos procedimientos genéricos, asumidos como una condición, se transforma en una fundación de la filosofía, es cuando se produce una "sutura" de ésta a alguno de aquellos campos.

Entonces, como afirma Alenka Zupancic en "The fifth condition" (2004), existiría una quinta condición de la filosofía, que apuntaría al cuidado de la distancia con respecto a sus condiciones. Proponemos una similar "quinta condición" (implícita en el riesgo de sutura), que sería también la que exige a la educación constituirse a cierta distancia respecto de los ámbitos productores de verdades. Por ejemplo, una educación política correría riesgo de sutura al convertirse en una educación adoctrinadora, una educación suturada al amor no podría desencadenar en otra cosa más que en un llano paternalismo o asistencialismo, una educación científica podría suturarse en un tecnicismo acrítico, y una enseñanza artística podría ser suturada a las formas sensibles y a la exaltación de las emociones.

La filosofía inventa una categoría de Verdad abstracta para poder pensar 
las diversas verdades de los diferentes campos en los que se establece su producción. Es decir, trabaja con las verdades a través de la mediación de la Verdad. La educación, en una ligera variación, se vincularía con las verdades para, a través del acto de pensamiento, extraer las consecuencias que éstas convocan en referencia a los saberes prestablecidos. En otras palabras, la educación se ubica en un paso intermedio entre la filosofía y los procedimientos de verdad, se ofrece permeable, en el campo del pensamiento, a las novedades acontecidas: dispone los saberes para que las verdades los atraviesen. La educación cuya exigencia es el pensamiento, parte del sitio en el que los saberes pueden descomponerse ante la aparición de una verdad, por ello se ubica, en cierta medida, en los límites interiores de cada campo. Si bien para Badiou la educación es principalmente interpretada como un "fragmento" de la política, destacamos la posibilidad de pensarla también como fragmento de los demás campos (científico, amoroso, artístico).

Tal como señalamos para el caso de la filosofía, la educación no es un conjunto de saberes cuya característica fundamental sea su transmisión a través de la explicación escolar, sino una actitud respecto de los saberes y las verdades, de lo establecido y de lo que acontece, de la repetición y de la creación, cuyo medio es el ejercicio colectivo del pensamiento. Como señalamos antes, Badiou asocia esta actitud con el gesto socrático que impulsa la crítica y la revuelta.

Tal es así, que en "El deseo de filosofía y el mundo contemporáneo" (2010), retoma el concepto de "revueltas lógicas" para describir a la filosofía. La dimensión de "revuelta" proviene de cierto descontento del pensamiento con el mundo establecido. Por el contrario, la educación explicadora obedece a la necesidad de la descripción técnica del mundo, a su fragmentación y especialización. De este modo, postula una "sociedad de la información", frecuentemente catalogada como un horizonte por diversos gobiernos, que trazan los criterios de evaluación que determinarán si una educación es de "buena" o "mala" calidad, de acuerdo al acceso universal con respecto a ese cúmulo de datos. Tal es el caso de los Informes para la Evaluación Internacional de los Estudiantes (PISA), basado en la cuantificación del rendimiento de los alumnos a través de exámenes estandarizados que miden la información incorporada para diversas áreas del saber, llevados a cabo por la OCDE (Organización para la Cooperación y el Desarrollo Económicos). En tal sentido, la educación por verdades parte de una toma de distancia 
con respecto al mundo (de allí su dimensión de "revuelta"), mientras que la educación estatal busca la inclusión o adaptación a ese mundo.

Otro aspecto señalado por Badiou para el caso de la filosofía, y que nosotros podríamos relacionar con la educación, es la propuesta de un proceso de ralentización: la invención de un tiempo para el pensamiento que, ante la demanda precipitada del mercado de los conocimientos, constituya un tiempo educativo propio. La educación del pensamiento se erige como una revuelta sosegada, en su afán de desplegar su potencia creativa. Su interés esencial no es tanto la incorporación de contenidos sino los procesos subjetivos, igualitarios y disruptivos (con respecto a los saberes) que puede desencadenar. Sin embargo, la propuesta de ralentización del proceso no impide la presura por la convocatoria a su propio tiempo.

Entiendo que el problema principal para el filósofo es llegar a tiempo. Esto lo pienso de verdad. Es la razón por la cual los filósofos perpetuamente tienen que dedicarse a las experiencias de su tiempo, tener un oído para lo que está ocurriendo, y escuchar tan atentamente al antifilósofo, puesto que lo que el antifilósofo les está diciendo continuamente es: 'Pero lo que tú estás discutiendo ya no existe más, o no existe en realidad, y además está esto por ejemplo de lo que tú no hablas y sin embargo es lo esencial', etc. Se trata de una convocatoria real al tiempo de uno. Es en verdad una carrera contra el tiempo. Estoy convencido de que los procedimientos de la verdad no esperan a la filosofia (BOSTEELS, 2003, p. 69).

El pensamiento tiene por exigencia estar dirigido hacia el tiempo presente. La educación, si es una auténtica educación por verdades, debe también considerar atentamente su propio tiempo, las experiencias de la actualidad. El saber, como señalamos, es la descripción del tiempo pasado. Todo acto de pensamiento, y en esto la educación no podría ser la excepción, debe atender, según Badiou, a las disrupciones acontecidas en el tiempo presente.

Siguiendo con el propósito de establecer un vínculo entre los conceptos de "pensamiento", "verdad", "filosofía" y "educación", aludiremos al Petit manuel d'inesthétique (1998), en el que Badiou aborda lateralmente algunas cuestiones educativas. En el texto, propone pensar la figura de un "nudo" entre arte, filosofía y educación. Y afirma que el arte "educa" únicamente por ser creador de verdades: 
$\mathrm{El}$ arte es educador simplemente porque produce verdades, y "educación" siempre ha querido decir (salvo en montajes opresivos o pervertidos) sólo esto: disponer los saberes de una forma tal que alguna verdad pueda agujerearlos. El arte educa por su sola existencia. No hay más que encontrar esa existencia, o en otras palabras: pensar un pensamiento (BADIOU, 1998, p. 54).

Como lo hemos venido sugiriendo, podríamos pensar, más allá de las distinciones explicitadas entre el arte y los otros campos (desarrolladas incluso en el propio texto), que así como el arte es "educador" por poseer la propiedad de producción de verdades, entonces la ciencia, la política y el amor, también podrían ser, de alguna manera, considerados como "educadores". La educación no produce verdades, pero los ámbitos de producción de verdades "educan".

Afirmamos antes que la educación se vinculaba con las verdades, de similar modo que la filosofía. Ahora bien, según la tesis de Badiou, la educación tendría un vínculo con las verdades de los diversos campos (por eso ellos "educan") y su tarea principal radicaría en permitir la permeabilidad en los saberes establecidos de las transformaciones y las novedades que imprimirían en ellos, a través del pensamiento, las actitudes propias desplegadas en cada ámbito: la invención científica, las creaciones artísticas, los nuevos vínculos amorosos y las producciones colectivas de igualdad. Por ello, la educación no podría ser reducida a la transmisión de contenidos, a la mera explicación, sino que debe ser experimentada y vivida junto con otros: "recordaremos que no existe educación sino por medio de verdades" (BADIOU, 1998, p. 60).

El pensamiento que es la educación hace posible el despliegue de un panorama contemporáneo, siempre actual, de las novedades producidas en el presente. No es casual que Badiou haya denominado sus cursos de un seminario impartido entre 2003 y 2004: "Images du temps présent". En él, desarrolla la existencia de un

conjunto de principios que posibilitan una visión del mundo determinada. Dichos principios se encuentran constituidos, principalmente, por un conjunto de declaraciones de existencia - en términos matemáticos serían axiomas o fundamentos - y de protocolos de acción que se derivan de ellos - protocolos de demostración u operaciones 
lógicas. A partir de estos componentes es posible construir diferentes discursos éticos y afectivos que legitiman intelectual y moralmente el mundo de lo posible y el de lo imposible, ya que el término se aplica también al mundo post-acontecimiental (BADIOU, 2003-2004).

Un encuentro educativo, en tanto singular incorporación a las verdades de una época definida, habilitaría la experiencia de que es factible vivir participando del proceso inventivo e inmanente de la producción contemporánea de una figura subjetiva. El individuo que se incorpora a estos procedimientos, se incorpora en realidad, a la construcción de un nuevo presente, mediante un cuerpo subjetivable (BADIOU, 2005-2006).

Hemos señalado que, mientras la filosofía favorece el encuentro con la Verdad o la Idea (aquí queda de manifiesto el retorno al platonismo propuesto por Badiou), la educación favorecería el encuentro con las verdades o las ideas propias de cada campo inventivo. Ahora bien, ese encuentro no asume la forma de la transmisión del saber, sino de la experimentación y el ejercicio colectivos. Asimismo, la transmisión de una experiencia, señala Badiou, supone además cierta vivencia del montaje formal en que esta experiencia es desplegada ${ }^{2}$, de allí la exigencia de la puesta en acto o de la participación activa de una potencia creadora más allá de la transmisión, y de la efectuación de ese acto educativo junto con otros, más allá de los procesos individuales de aprendizaje: estos requerimientos no son otra cosa más que la exigencia de pensar un sujeto colectivo para la educación.

\section{Referências}

BADIOU, Alain. Conditions. París: Seuil, 1992.

. El deseo de filosofía y el mundo contemporáneo. In: La filosofía, otra vez. Madrid: Errata naturae, 2010.

. Images du temps présent, Entretemps. n. 3, 2003-2004. Disponível em: $<$ http://www.entretemps.asso.fr/Badiou/03-04.3.htm>. Acesso em: 07 Jul. 2013.

2 Esta idea aparece con insistencia en las intervenciones de Alain Badiou, puede hallarse implícito en Petit manuel d'inesthétique (1998), y más específicamente en Bosteels, Posmaoísmo: un diálogo con Alain Badiou (2003). 
. L'être et l'événement. París: Seuil, 1988.

. La confesión del filósofo, Reflexiones marginales, ano 3, n. 15, jun./jul., 2013. Disponível em: $<$ http://reflexionesmarginales.com/3.0/19-laconfesion-del-filosofo/>. Acesso em: 07 Jan. 2014.

. Logiques des mondes. L'être et l'événement, 2. París: Seuil, 2006. . Petit manuel d'inesthétique. París: Seuil, 1998.

. Philosophy as creative repetition. The Symptom, n. 8, Winter, 2007.

. S'orienter dans la pensée, s'orienter dans l'existence, Entretemps, n. 2, 2005-2006. Disponível em: <http://www.entretemps.asso.fr/Badiou/ 05-06.2.htm>. Acesso em: 09 Jul. 2013.

BARTLETT, A. Badiou and Plato. An education by truths. Edinburgh: University Press, 2011.

. Conditional notes on a New Republic. En: ASHTON, P.; BARTLETT, A.; CLEMENS, J. The praxis of Alain Badiou. Melbourne: Repress, 2006a.

. The pedagogical theme: Alain Badiou and an eventless education. Anti-Thesis, n. 16, $2006 \mathrm{~b}$.

BERGEN, V. Pensée et être chez Deleuze et Badiou (Badiou lecteur de Deleuze). In: RAMOND, C. Alain Badiou. Penser le multiple. París: L'Harmattan, 2002.

BOSTEELS, B. Posmaoísmo, un dialogo con Alain Badiou. Revista Acontecimiento, n. 24-25, p. 45-78, 2003.

. Vérité et forçage: Badiou avec Heidegger et Lacan. In: RAMOND, C. Alain Badiou. Penser le multiple. París: L'Harmattan, 2002.

ZUPANCIC, A. The fifth condition. In: HALLWARD, P. Think again: Alain Badiou and the future of philosophy. New York: Continuum, 2004.

Data de registro: 06/03/2014

Data de aceite: 22/03/2015 